\title{
Mass Spectrometry of Oligonucleotides
}

\author{
Selina T.M. Monn, Jan M. Tromp, and Stefan Schürch*
}

\begin{abstract}
Within the past decade mass spectrometry has undoubtedly consolidated its role as a major player in modern bioanalysis. Mass spectrometry of oligonucleotides is an evolving field that has not reached its culmination point yet, as many aspects of oligonucleotide dissociation in gas phase still need to be clarified. Research in our group is focused on the elucidation of the dissociation mechanisms of oligonucleotides and their analogs and on the characterization of metal-oligonucleotide complexes by tandem mass spectrometry in order to gain structural information. Here, we present the dissociation mechanism of RNA and an example for the characterization of metal-oligonucleotide complexes.
\end{abstract}

Keywords: Collision-induced dissociation · Iron(III) - Metal-oligonucleotide complexes · RNA ·

Tandem mass spectrometry

\section{Introduction}

Modified oligonucleotides play an important role in many disciplines of life sciences and medicine. Antisense oligonucleotides find application in human therapy or as genetic probes for diagnostic purposes in molecular biology. Aptamers exhibiting catalytic activity towards specific protein targets are another family of nucleic acid based therapeutics. Besides the enhancement of binding properties and biostability by the introduction of structural modifications, the physicochemical properties of oligonucleotides are influenced by incorporation of metal ions. Metal ions are important cofactors stabilizing oligonucleotide folding whereas certain organo-metallic

${ }^{*}$ Correspondence: Dr. S. Schürch

Department of Chemistry and Biochemistry

University of Bern

Freiestrasse 3

$\mathrm{CH}-3012$ Bern

Tel.: +41316314389

Fax: +41316313421

E-Mail: stefan.schuerch@ioc.unibe.ch

http://www.dcb.unibe.ch/groups/schuerch/ compounds act as potent anti-tumor agents Specific binding of metal ions to biomolecules bears a great potential for analytical purposes, as it affects the gas-phase behavior of oligonucleotides, thus providing complementary tandem mass spectrometric data.

Expanding research in the field of modified oligonucleotides demands suitable analytical tools for size and purity verification of known compounds and accurate structure elucidation of unknowns. There is a need to characterize the types and sites of modifications in oligonucleotides and to identify and sequence selected candidates originating from combinatorial syntheses.

\section{Sequence Determination of Oligoribonucleotides (RNA) by Tandem Mass Spectrometry}

Due to the unnatural structural elements present in antisense oligonucleotides, the classical techniques for sequence determination are likely to fail and alternative analytical tools are needed. Tandem mass spectrometry is a highly promising candidate for this task, as the method provides detailed structural information due to the structure-specific fragment ions formed upon collision-induced dissociation (CID), while requiring minute quantities of sample only.

In contrast to the automated mass spectrometric analysis of peptides and proteins, which is routinely applied in many laboratories, sequence determination of oligonucleotides by tandem mass spectrometry is hardly employed. When we started to ad- dress this topic a few years ago, we faced highly complex analytical data generated by tandem mass spectrometry of even short nucleotide sequences. The structural variations present in antisense oligonucleotides affect the dissociation mechanism along with the cleavage sites and consequently render the interpretation of analytical data difficult. To establish a reliable protocol for oligonucleotide sequencing based on tandem mass spectrometry, the fundamental mechanistic aspects of oligonucleotide dissociation in gas-phase have to be elucidated in detail.

While dissociation of oligodeoxyribonucleotides (DNA) in gas-phase has been investigated by several groups over the past decade [1-3], hardly any conclusive data on the dissociation of oligoribonucleotides (RNA) and modified oligonucleotides are found in literature. In contrast to published data on DNA, which shows that DNA predominantly dissociates into [a-B]- and wions due to cleavage of the 3 '-P-O bond, we found the preferred cleavage site of the RNA backbone to be the 5'-P-O bond, resulting in abundant c- and y-ions [4]. Examples of DNA and RNA typical product ion spectra, along with the nomenclature of the fragment ions, are shown in Fig. 1. Furthermore, our data demonstrated that backbone dissociation of mixed-sequence DNA/RNA-oligomers is locally controlled and influenced by the adjacent ribose $2^{\prime}$ substituent only. Since the ribose 2 '-substituent is the only structural variation between the DNA and RNA sequences investigated, it must be responsible for the altered dissociation behavior of the two types of oligonucleotides. 


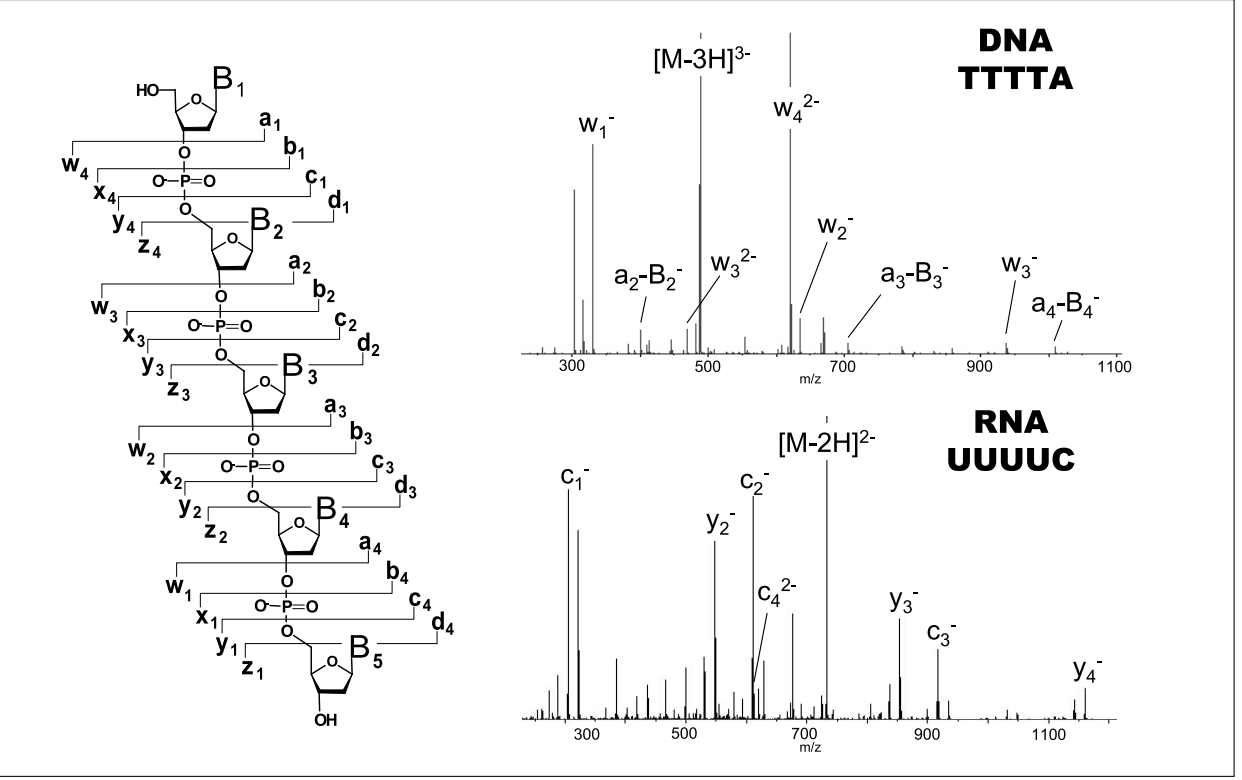

Fig. 1. Product ion spectra of $d(T T T T A)$ and UUUUC. The spectrum of DNA is characterized by [a-B]and w-fragment ions, whereas RNA primarily dissociates into c- and y-ions. The scheme shows the nomenclature of oligonucleotide fragments, as proposed by McLuckey et al. [1]. a

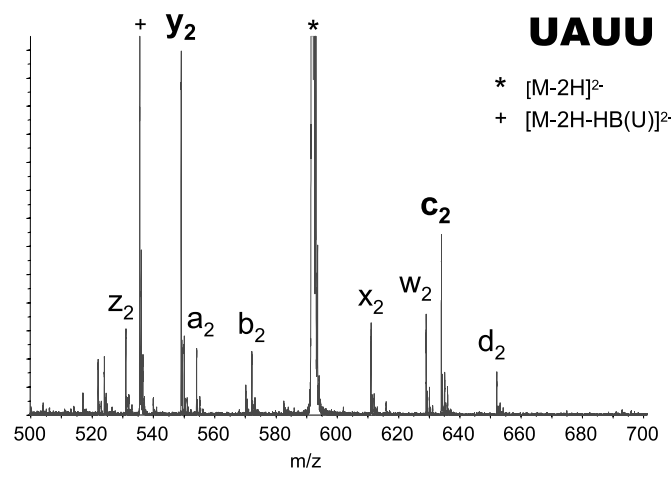

b

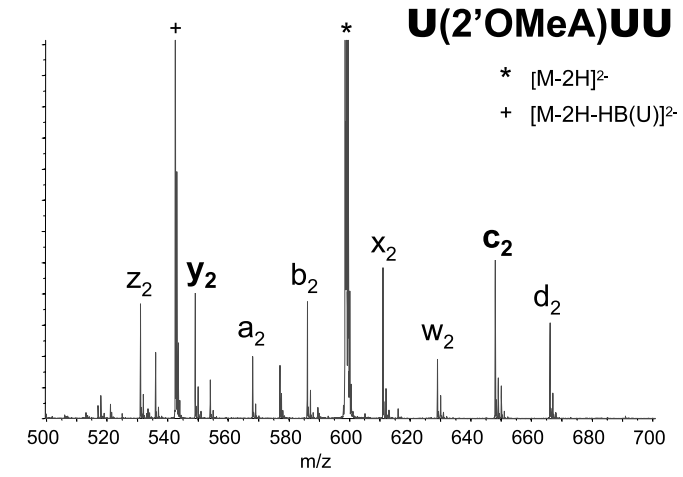

C

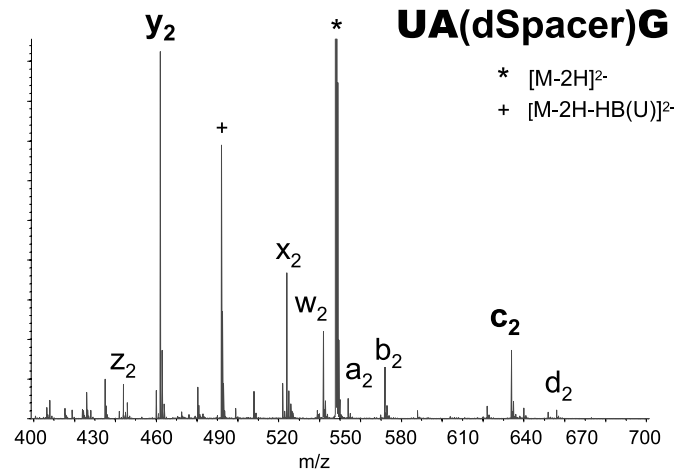

The mechanism of RNA dissociation in gas-phase was elucidated by studying the influence of various $2^{\prime}$-substituents and nucleobases on backbone cleavage, as both groups can potentially participate in the proton transfer that is involved in the dissociation mechanism of RNA. Fig. 2a shows a section of the product ion spectrum of the doubly deprotonated tetraribonucleotide UAUU ( $\mathrm{m} / \mathrm{z}$ 591.63). The abundant $\mathrm{c}-$ and $\mathrm{y}$-ions confirm the $5^{\prime}-\mathrm{P}-\mathrm{O}$ bond as the preferred cleavage site of the RNA backbone. The picture changes when the 2 '-hydroxyl group is replaced by a methoxy substituent. As demonstrated by the product ion spectrum of $\mathrm{U}\left(2^{\prime} \mathrm{OMeA}\right) \mathrm{UU}\left([\mathrm{M}-2 \mathrm{H}]^{2-}, \mathrm{m} / \mathrm{z}\right.$ 598.60), the lack of the transferable proton inhibits the RNA typical dissociation pathway, resulting in all of the theoretically possible fragment ions to be generated with similar abundance (Fig. 2b). Experiments on oligoribonucleotides incorporating 2 '-modifications demonstrated, that the availability of a transferable 2 '-proton in the vicinity of the phosphodiester group contributes to the formation of the RNA typical c- and y-ions. Such behavior of RNA is opposed to the backbone dissociation of DNA, which is initiated by protonation and loss of the nucleobase.

We propose a gas-phase dissociation mechanism, where backbone cleavage is initiated by formation of an intramolecular cyclic transition state with the 2 '-hydroxyl proton bridged to the $5^{\prime}$-phosphate oxygen [5]. Transfer of the $2^{\prime}$-hydroxyl proton to the $5^{\prime}$-oxygen leads to dissociation of the $5^{\prime}$-P-O bond and finally, complementary cand y-ions are released. Subsequent bond rearrangement leads to stable c-ions (Fig. 3a). A dissociation mechanism similar to the one known for backbone dissociation of oligoribonucleotides in solution (Fig. 3b) might also be feasible in gas-phase, as it involves the 2'-hydroxy proton and leads to the same RNA typical c- and y-ions as well. To evaluate such alternative mechanism, the tetranucleotide UA(dSpacer) $\mathrm{G}$, incorporating a building block lacking the nucleobase, has been subjected to MS/MS analysis. The modification allows the evaluation of the role of the nucleobase in the dissociation mechanism, since there is no nucleobase which could potentially be involved in a proton transfer. A section of the product ion spectrum of $\mathrm{UA}(\mathrm{dSpacer}) \mathrm{G}$, reflecting the fragment ions generated by cleavage of the
Fig. 2. Product ion spectra of RNA and modified tetraribonucleotides. The $[\mathrm{M}-2 \mathrm{H}]^{2-}$ ion was selected as the precursor for collision-induced dissociation. a) Spectrum of the unmodified tetraribonucleotide UAUU, showing the cand y-ions as the most abundant fragments. b) Spectrum of U(2'OMeA)UU, which lacks the transferable 2'-proton of adenosine. The absence of any preferred type of fragment ion points out the key role of the 2'-hydroxy proton of RNA. c) Spectrum of UA(dSpacer)G, incorporating an abasic site as the third building block. Abundant $\mathrm{C}_{2}$ - and $\mathrm{y}_{2}$-fragment ions are generated, despite the lack of the adjacent nucleobase. 
phosphodiester adjacent to the dSpacer, is shown in Fig. 2c. The $y_{2}$-fragment ion is observed with more than threefold abundance of the other 3 '-terminal fragment ions and the $c_{2}$-ion is the most abundant $5^{\prime}$-terminal one. The observed RNA typical fragment ion pattern demonstrates that the nucleobase is not involved in the dissociation mechanism of the phosphodiester backbone. The experiment confirms the dissociation mechanism given in Fig. 3a and an alternative gas-phase dissociation mechanism similar the one known for cleavage of oligoribonucleotides in solution can be rejected.

Tandem mass spectrometry does provide the accuracy, sensitivity and speed needed for characterization of this biologically significant class of compounds. However, increased understanding of the gas-phase behavior of natural and modified oligonucleotides is needed to take full advantage of the capabilities of modern analytical instrumentation for structure elucidation.

\section{Mass Spectrometry of Metal- Oligodeoxynucleotide Complexes}

Exploration of metal-oligodeoxynucleotide complexes by tandem mass spectrometry is a topic that is rarely investigated but which holds great potential for future applications [6]. Pentadeoxynucleotides have been chosen as model compounds to elucidate the fragmentation pattern of metal-oligonucleotide complexes [7]. While oligonucleotides are commonly analyzed in the negative ion mode, charge compensation caused by complexation of metal cations

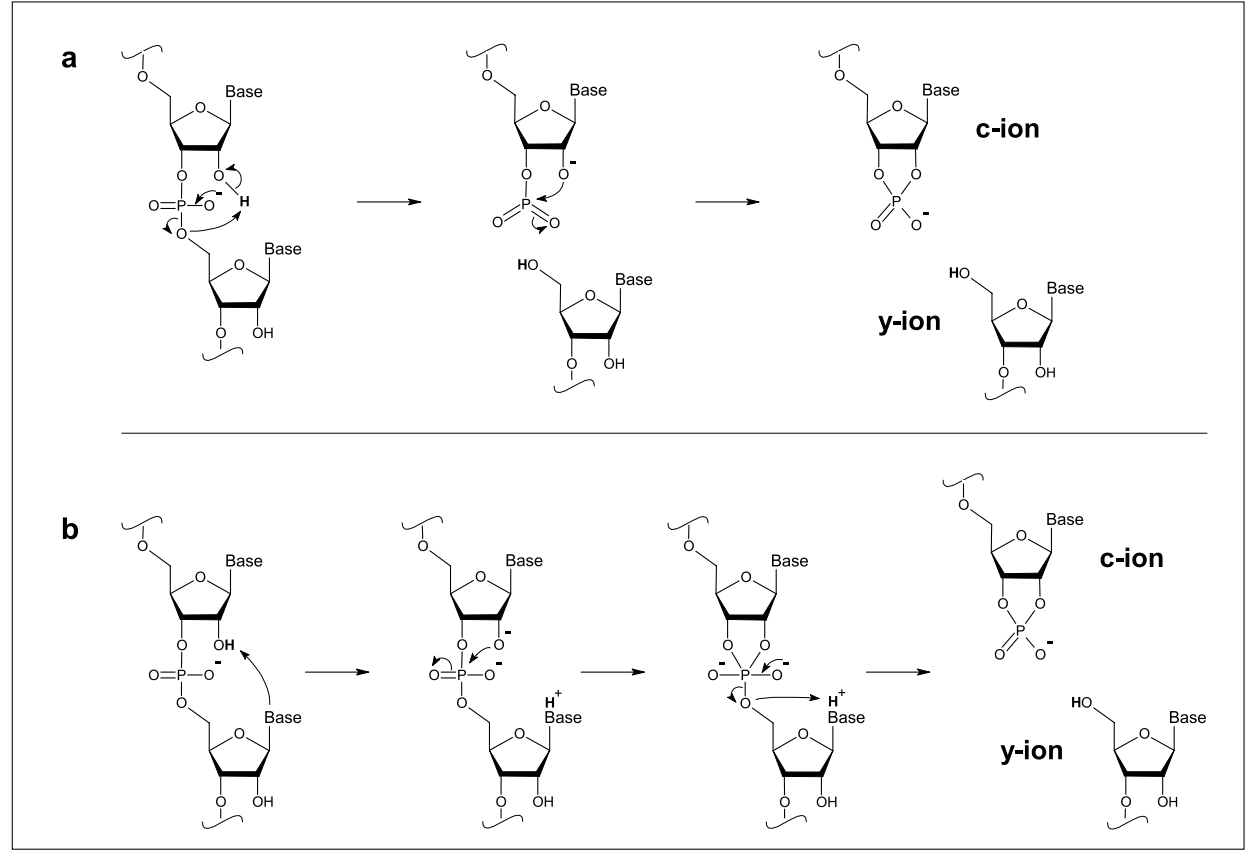

Fig. 3. a) Proposed mechanism for the gas-phase dissociation of RNA, resulting in c-and y-fragment ions. b) The alternative dissociation mechanism involving the nucleobase as a proton acceptor could be rejected.

renders analysis in the positive ion mode more favorable. Our studies demonstrated that the fragmentation of uncomplexed protonated oligodeoxynucleotides does not differ significantly from the fragmentation pattern of deprotonated oligodeoxynucleotides, mainly resulting in the $\mathrm{w}$ - and (a-B)fragment ions typical of DNA. These observations are in agreement with the published dissociation mechanism of DNA, which assumes protonation of the nucleobase as the first step initiating subsequent base loss and backbone cleavage [3].

Our experiments revealed a striking difference between the product ion spectra of iron(III)-pentamer complexes and the spectra of uncomplexed oligonucleotides. Abundant peaks due to $\mathrm{Fe}(\mathrm{III})$-complexed $[\mathrm{M}-\mathrm{T}]-, \mathrm{w}_{4^{-}}$, and $\left[\mathrm{w}_{4}-\mathrm{B}\right]-$ fragment ions are observed. Additionally, the internal $\left[\mathrm{w}_{4}\right.$ $\mathrm{d}_{4}$ ]-fragment ion is detected (Fig. 4). All fragment ions of high abundance are metal-

\section{TTGAT}

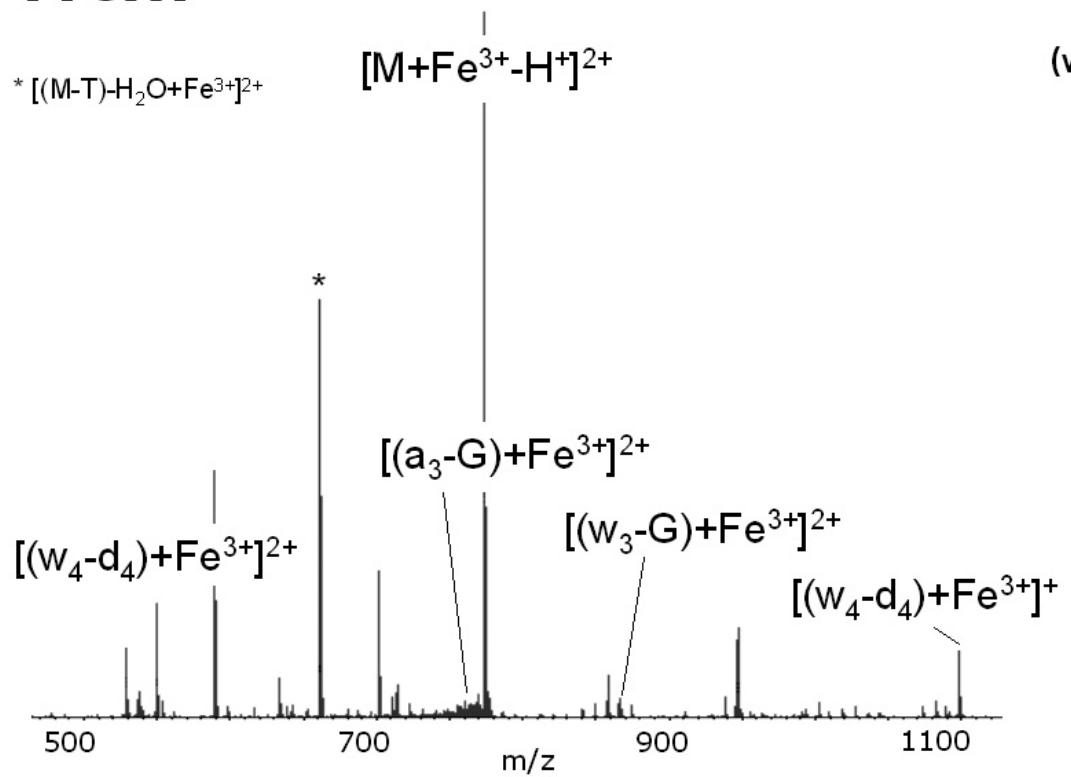

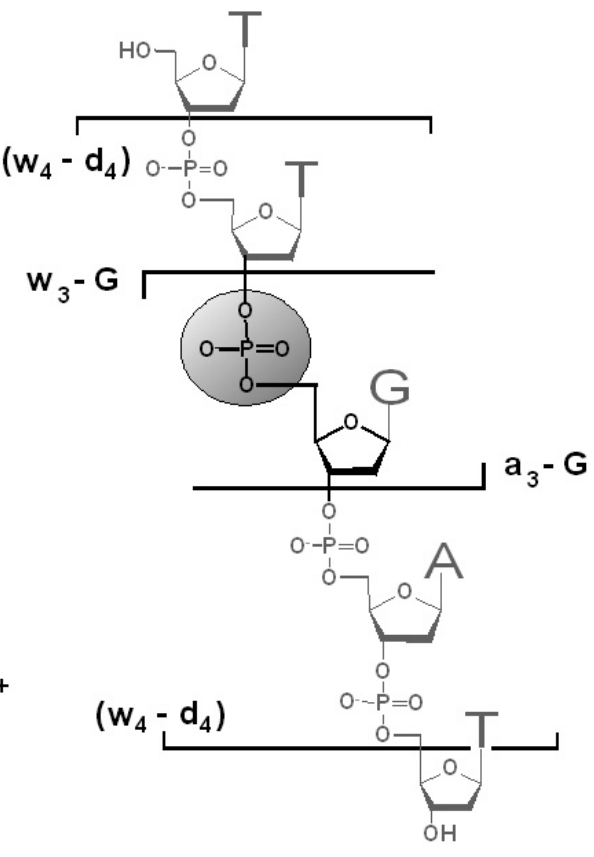

Fig. 4. Successful localization of the $\mathrm{Fe}\left(\right.$ (III) ion demonstrated for the pentadeoxynucleotide $\mathrm{d}(\mathrm{TTGAT})$. The $\left[\mathrm{M}+\mathrm{Fe}^{3+}-\mathrm{H}^{+}\right]^{2+}$ ion was selected as the precursor ion. Key fragments providing information about the coordination site are indicated in the spectrum. 
complexed, whereas small fragments, e.g. $\mathrm{w}_{2}$, remain uncomplexed and are observed with very low abundance. These results strongly suggest a very tight bond between the Fe(III)-ion and the oligonucleotides. Furthermore, the $\mathrm{Fe}(\mathrm{III})$-ion has a stabilizing effect on the central region of the oligonucleotide, as the $\left[\mathrm{w}_{4}-\mathrm{d}_{4}\right]$ core fragment is observed with high abundance. Apparently, it is not feasible to separate the Fe(III)-ion from the oligonucleotide under low-energy CID conditions.

Occurrence or absence of certain metalcomplexed fragment ions provides information about the site of complexation [7]. Other authors suspected the nucleobases as the binding sites. However, considering the high abundance of metal-complexed fragments with cleaved nucleobase, like $\left[\mathrm{w}_{4}-\mathrm{B}\right]$, we cannot support this assumption. In our approach, localization of the binding site of the Fe(III)-ion was performed by identification of the shortest metal-complexed fragment ions from the $3{ }^{\prime}$ - and the $5^{\prime}$-end (Fig. $4)$. Simultaneous occurrence of the metalcomplexed fragment ions $\left[\mathrm{w}_{3}-\mathrm{B}_{3}\right]$ and $\left[\mathrm{a}_{3}-\right.$ $\mathrm{B}_{3}$ ] restricts the position of the $\mathrm{Fe}$ (III)-ion to the third nucleotide from the 3 '-end. Since the non-terminal sugar residues do not pro- vide any suitable binding site for metal ions, the $\mathrm{Fe}(\mathrm{III})$-ion must coordinate to the third nucleotide. Coordination of the nucleobase can clearly be excluded, as both decisive fragment ions lack the nucleobase. Analyses of various $\mathrm{Fe}(\mathrm{III})$-pentamer complexes gave identical results and the $\mathrm{Fe}$ (III)-ion can undoubtedly be assigned to the second phosphate group from the $5^{\prime}$-end (Fig. 4).

Extended studies on $[\mathrm{Fe}(\mathrm{II})]_{3}$-pentamer and $[\mathrm{Zn}(\mathrm{II})]_{3}$-pentamer complexes revealed very strong complexation as well, as no metal ions were released upon CID. Iron(III), iron(II) and zinc(II) ions all form extremely stable complexes with oligonucleotides and induce a strong stabilization of the phosphate backbone, thus resulting in greatly reduced fragmentation.

Tandem mass spectrometry provides insight into the complex world of metaloligonucleotide complexes. The technique holds a great potential for characterization of naturally occurring complexes, localization of metal ions, and even investigation of metal ion induced conformational changes. Since the addition of metal ions affects the gas-phase behavior of oligonucleotides, it provides an alternative route for gaining sequence information.

\section{Acknowledgments}

We wish to thank the Swiss National Science Foundation for financial support of this work.

Received: August 17, 2005

[1] S.A. McLuckey, G.J. Van Berkel, G.L. Glish, J. Am. Soc. Mass Spectrom. 1992, 3, 60-70.

[2] E. Nordhoff, F. Kirpekar, P. Roepstorff, Mass Spectrom. Rev. 1996, 15, 67-138.

[3] Z. Wang, K.X. Wan, R. Ramanathan, J.S Taylor, M.L. Gross, J. Am. Soc. Mass Spectrom. 1998, 9, 683-691.

[4] S. Schürch, E. Bernal-Méndez, C.J. Leumann, J. Am. Soc. Mass Spectrom. 2002 13, 936-945.

[5] J.M. Tromp, S. Schürch, J. Am. Soc. Mass Spectrom. 2005, 16, 1262-1268.

[6] J.L. Beck, M.L. Colgrave, S.F. Ralph, M.M. Sheil, Mass Spectrom. Rev. 2001, 20, 61-87.

[7] S.T.M. Monn, S. Schürch, J. Am. Soc Mass Spectrom. 2005, 16, 370-378. 